\title{
Coração artificial pneumático: seleção de materiais, fabricação de protótipo e ensaios hidrodinâmicos
}

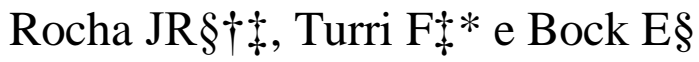 \\ §Instituto Federal de Educação Ciência e Tecnologia de São Paulo - IFSP. †Faculdade de \\ Tecnologia de São Paulo FATEC SP †Instituto de Ensino e Pesquisa - INSPER *Laboratório de \\ Pneumologia Experimental da Faculdade de Medicina - USP.
}

\begin{abstract}
Resumo. O número de transplantes de coração cresce anualmente em torno de $60 \%$ devido a problemas de saúde que vêm assolando a população mundial. E, devido à falta de órgãos disponíveis, a fila de espera aumenta podendo chegar a meses. Colaborando com esse cenário desolador, há o agravamento da saúde das pessoas com a evolução da insuficiência cardíaca. Com o avanço da ciência e das tecnologias disponíveis, foram desenvolvidos dispositivos para ajudar ou melhorar a qualidade de vida desses pacientes, chamados de dispositivos de assistência ventricular, como é o caso das bombas de sangue e do coração artificial. Este trabalho visa o levantamento bibliográfico, projeto e fabricação de um dispositivo desde sua concepção, seleção de materiais e a fabricação. A metodologia de avaliação foi baseada em ensaios de desempenho hidrodinâmico com fluido simulando o sangue em um circuito fechado. Os resultados preliminares apontam para uma alternativa promissora para o mercado desse tipo de dispositivo com tecnologia nacional e competitiva. Em trabalhos futuros, o coração artificial será avaliado em ensaios com "in vivo" em animais e ensaios clínicos.
\end{abstract}

Palavras-Chave. Coração artificial, Fabricação de protótipo, Dispositivos de Assistência Ventricular, Transplante Cardíaco e Biomateriais.

\section{Pneumatic artificial heart: materials selection, prototype, manufacturing and hydrodynamic tests}

\begin{abstract}
The number of heart transplants grows around $60 \%$ annually due to health problems that have been plaguing the world population. And, due to the lack of available organs, the waiting list increases and can reach months. Collaborating with this bleak scenario, there is a worsening of people's health with the evolution of heart failure. With the advancement of science
\end{abstract}


and available technologies, devices have been developed to help or improve the quality of life of these patients, called ventricular assist devices, such as blood pumps and the artificial heart. This work aims at the bibliographical survey, design and manufacture of a device from its conception, selection of materials and manufacture. The evaluation methodology was based on hydrodynamic performance tests with fluid simulating blood in a closed circuit. The preliminary results point to a promising alternative for the market of this type of device with national and competitive technology. In future work, the artificial heart will be evaluated in trials with "in vivo" in animals and clinical trials.

Keyworks: Artificial heart, Prototype manufacturing, Ventricular Assist Devices, Heart Transplant and Biomaterials

Introdução. As doenças relacionadas ao coração são responsáveis pela a causa principal de mortes mundialmente. Estima-se que 17,7 milhões de pessoas morreram por doenças cardiovasculares e, 2015, representando 31\% da população mundial. Destes óbitos, 7,4 milhões ocorrem devido a doenças cardiovasculares e 6,7 milhões, decorrentes a acidentes vasculares cerebrais (AVC). E ainda para agravar o problema, mais de três quartos das mortes por doença cardiovasculares, ocorrem em países de baixa e média renda. No Brasil, até em 2017 as mortes relacionadas ao coração em torno de 380 mil pessoas que perderam suas vidas [1-3].

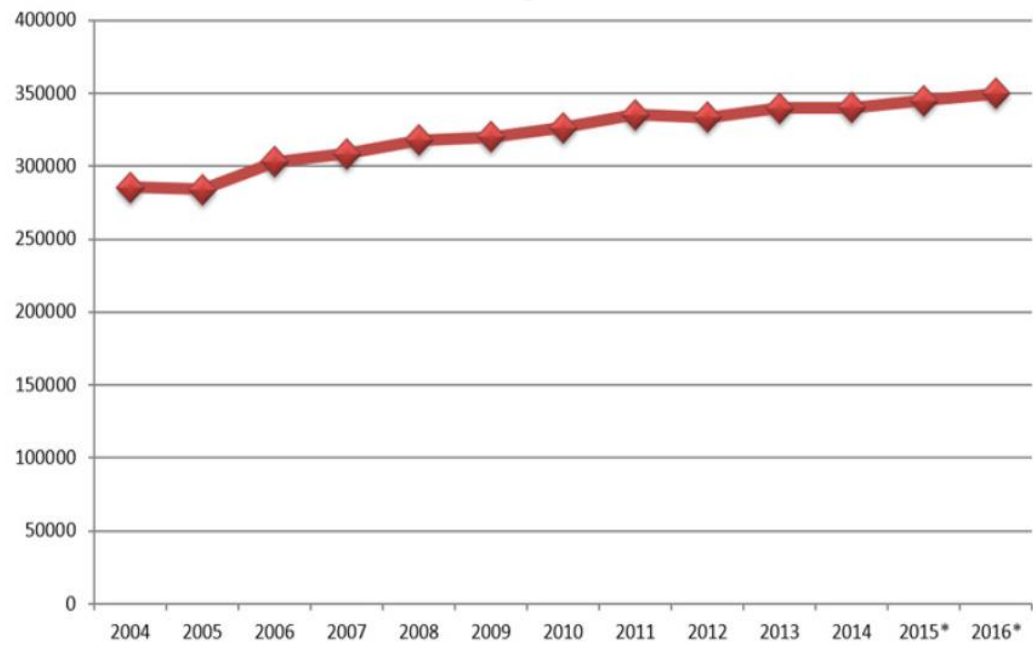

Figura 1 - Mortes relacionadas a doenças cardiovasculares. Fonte: Incor 
Até aqui, segundo o Instituto do Coração do Hospital das Clínicas da USP (Incor), foram realizados 1080 procedimentos. Desde o primeiro, em 1985, foram 230 transplantes de coração. Até aqui, segundo o Instituto do Coração do Hospital das Clínicas da USP (Incor), foram realizados 1080 procedimentos. Desde o primeiro, em 1985, foram 230 transplantes de coração em crianças e 564 em adultos. Estes números correspondem a $40 \%$ de todos os procedimentos de transplantes de coração realizados no estado de São Paulo e 37\% no Brasil [3-5].

O coração é um órgão presente na vida humana e animal e tem a função de bombear o sangue através dos vasos sanguíneos do sistema circulatório, levando o oxigênio e nutrientes e eliminando os resíduos produzidos metabolicamente. Este órgão é involuntário e independente de qualquer comando e dividido em quatro cavidades. Na parte superior situam-se as aurículas direita e esquerda e, na parte inferior, os ventrículos direito e esquerdo [1, 3].

Funcionamento do coração. Já define que o coração é um órgão muscular que se contrai ritmicamente, enquanto bombeia o sangue pelo o sistema circulatório. Também é responsável pela produção de um hormônio chamado de fator natriurético atrial. Suas paredes são constituídas de três túnicas: a interna, ou endocárdio; a media, ou miocárdio; e a externa, ou pericárdio. A região central fibrosa do coração, comumente chamado de esqueleto fibroso, serve de ponto de apoio para as válvulas, além de ser o local de origem e inserção de células musculares cardíacas [1].

O coração adulto médio bombeia o sangue numa frequência de 60 a 100 batimentos por minutos (bpm) e possui duas etapas que contrai durante a execução do batimento [1-3]:

1. Na primeira etapa, os átrios direito e esquerdo contraem-se ao mesmo tempo, bombeando sangue para os ventrículos direito e esquerdo;

2. Na segunda etapa, os ventrículos contraem -se juntos para retirar o sangue do coração. $\mathrm{O}$ músculo cardíaco então relaxa antes do próximo batimento, isto permite que o coração se encha de sangue novamente. 


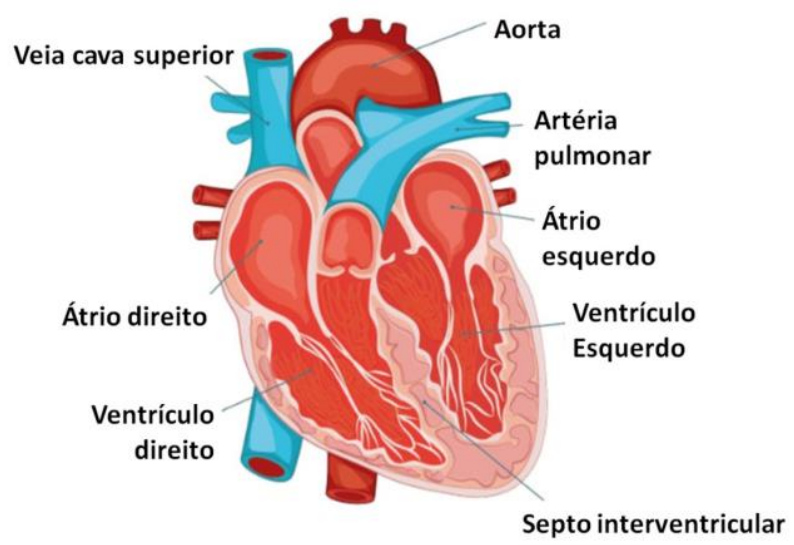

Figura 2 - Câmaras e principais vasos mostrando a circulação interna do coração.

Dispositivo de assistência ventricular. $O$ coração artificial, também conhecido como dispositivo de assistência ventricular (DAV) é capaz de servir dando um suporte ao paciênte que aguarda por um novo coração. Estes dispositivos servem tanto para pacientes adultos como pediátricos, ajudando a melhorar a qualidade de vida por meses. Assim pacientes acometidos por doenças como cardiomiopatia restritiva, estes são os maiores beneficiados [4-10].

Este dispositivo possui duas câmaras separadas por uma membrana. Uma das câmaras é a passagem de ar comprimido, onde é conectado a uma conexão, assim promovendo o movimento da membrana. Já a outra câmara, promove a circulação do sangue, onde temos duas passagens, a entrada e a saída do sangue. Este processo é controlado por software, onde há a possibilidade de ajustar a frequência dos batimentos, portanto podemos melhorar o volume e a eficiência de contração $[3,5,10]$.

Os dispositivos que são implantados do lado externo do paciente são chamados de paracórporeos e ajudão o coração a bombear o sangue [1]. 


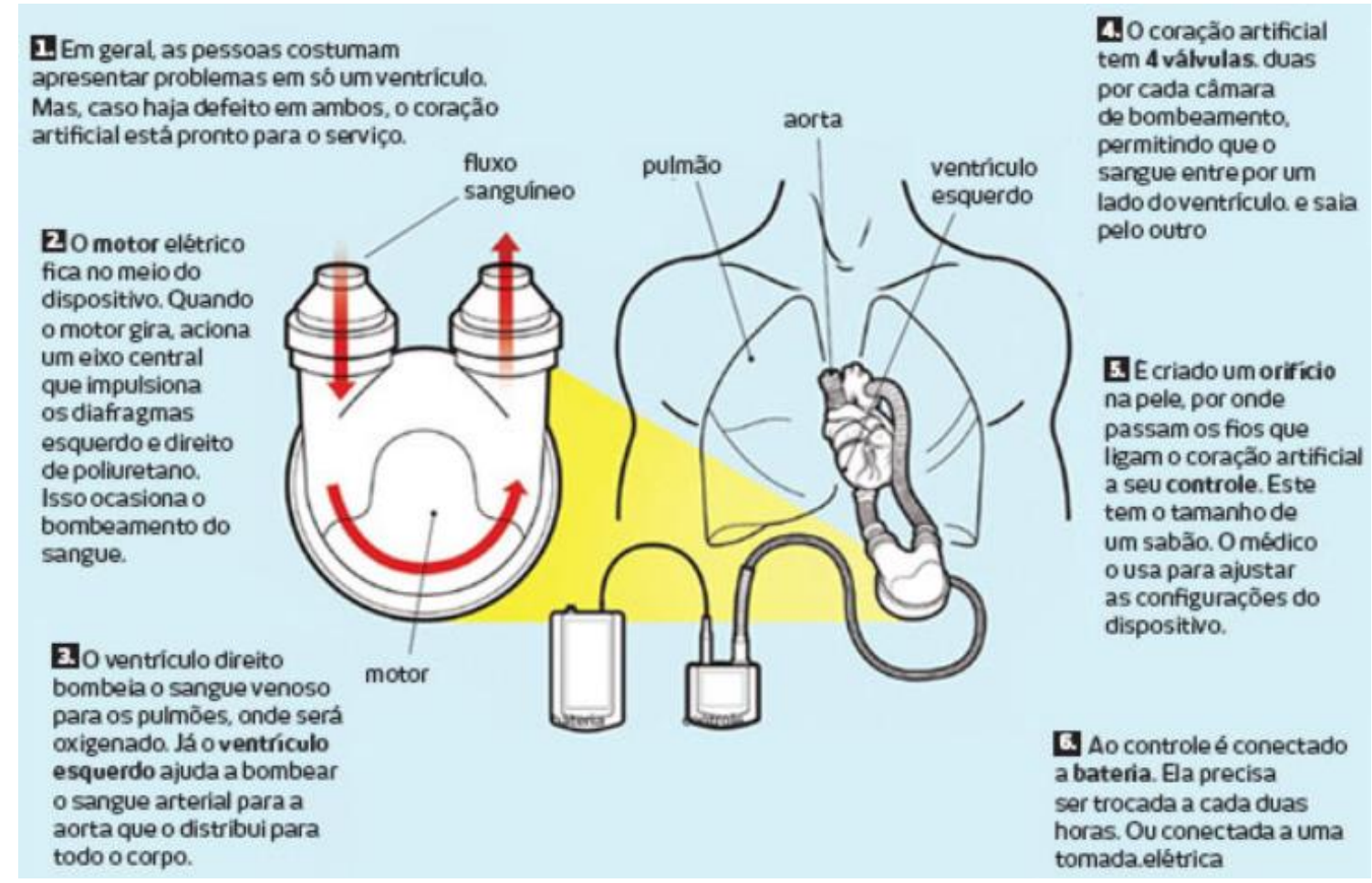

Figura 3 - Funcionamento do Coração Artificial Auxiliar CAA - IDPC [1].

Seleção de materiais e manufatura. Os biomateriais são empregados em componentes/dispositivos implantados interior no corpo humano ou em animais e têm a finalidade de substituir partes do corpo doentes ou danificadas. Estes materiais não podem ter ou produzir substâncias nocivas à saúde e devem ser compatíveis com os tecidos do corpo. Podemos ter biomateriais como os metais, poliméricos, cerâmicos, compósitos e semicondutores [6-9].

Para aplicar um determinado material, deve-se conhecer as propriedades como a mecânica, elétrica, térmica, magnética, ótica, e deteriorativa além disso, os quatro componentes que são importantes para garantir um bom funcionamento que são: Processamento, Estrutura, Propriedades e Desempenho [8,9].

Durante a usinagem deve-se ter as boas práticas de produção para evitar problemas durante a fabricação, por exemplo os cantos vivos de peças, roscas e elementos durante a montagem. Para isso é recomendado a aplicação de raios e chanfros, assim eliminando tensões e prováveis problemas durante o manuseio.

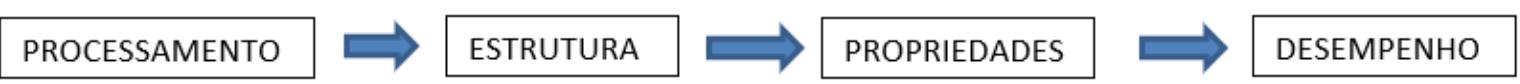


Para a fabricação do coração artificial, foi utilizado aço inoxidável 316L e o titânio G2 para testes e simulações mais próximos dos dispositivos implantados em pessoas. A ilustração a seguir, descreve o conjunto de peças fabricadas e materiais aplicados. Todas as peças usinadas foram fabricadas no Torno Romi CNC Centur 30D, para garantir qualidade e boa rugosidade no produto final[6].

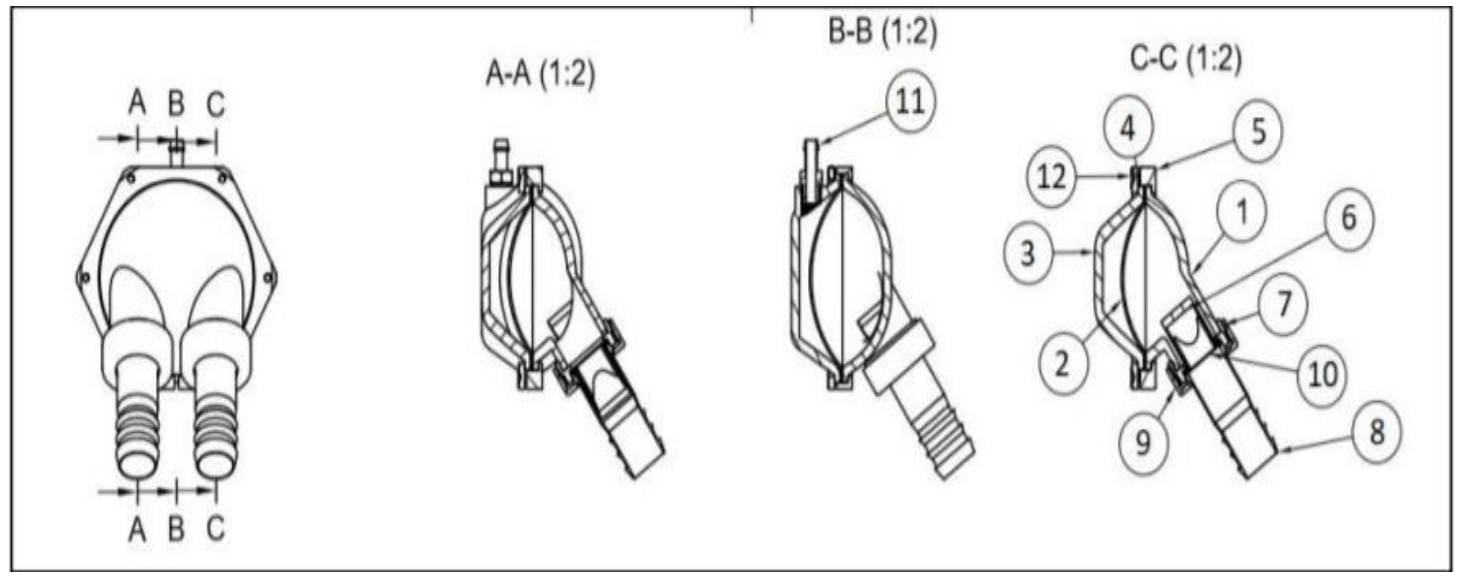

Figura 4- Vistas do projeto e especificações na tabela 1.

Tabela 1 - Componentes do DAV.

\begin{tabular}{|c|c|c|c|}
\hline \multicolumn{5}{|c|}{ PROTÓTIPO - CORAÇÃO ARTIFICIAL } \\
\hline ITEM & QUANTIDADE & DESCRIÇÃo & MATERIAL \\
\hline 1 & 1 & Carcaça & Resina 3D \\
\hline 2 & 1 & Membrana & Poliuretano \\
\hline 3 & 1 & Tampa & Resina 3D \\
\hline 4 & 1 & Anel da Tampa & Aço Inoxidável 316L \\
\hline 5 & 1 & Trava & Aço Inoxidável 316L \\
\hline 6 & 2 & Válvula & Poliuretano \\
\hline 7 & 2 & Inserto Metálico & Titânio G2 \\
\hline 8 & 2 & Conexão Entrada-Saída & Titânio G2 \\
\hline 9 & 2 & Porca Conexão Entrada-Saída & Titânio G2 \\
\hline 10 & 2 & Anel da Válvula & Resina 3D \\
\hline 11 & 1 & Conexão Linha Pneumática & Aço Inoxidável 316L \\
\hline 12 & 6 & Parafusos & Aço Inoxidável 316L \\
\hline
\end{tabular}




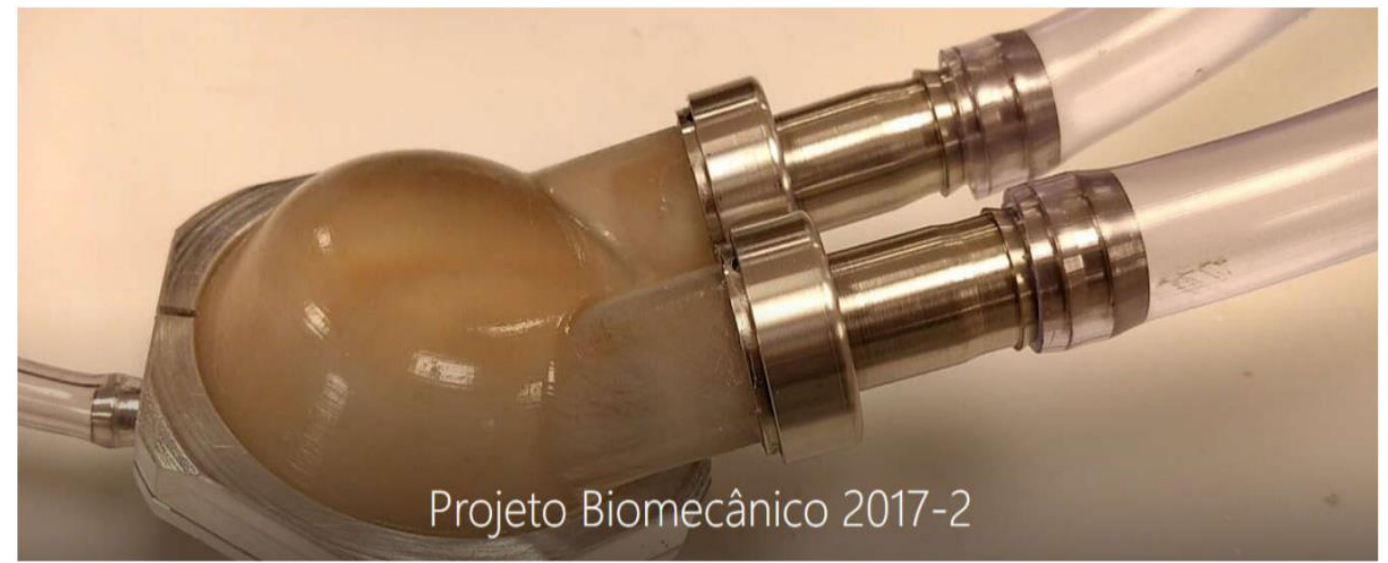

Figura 5 -Dispositivo fabricado. Fonte: TechLab Insper.

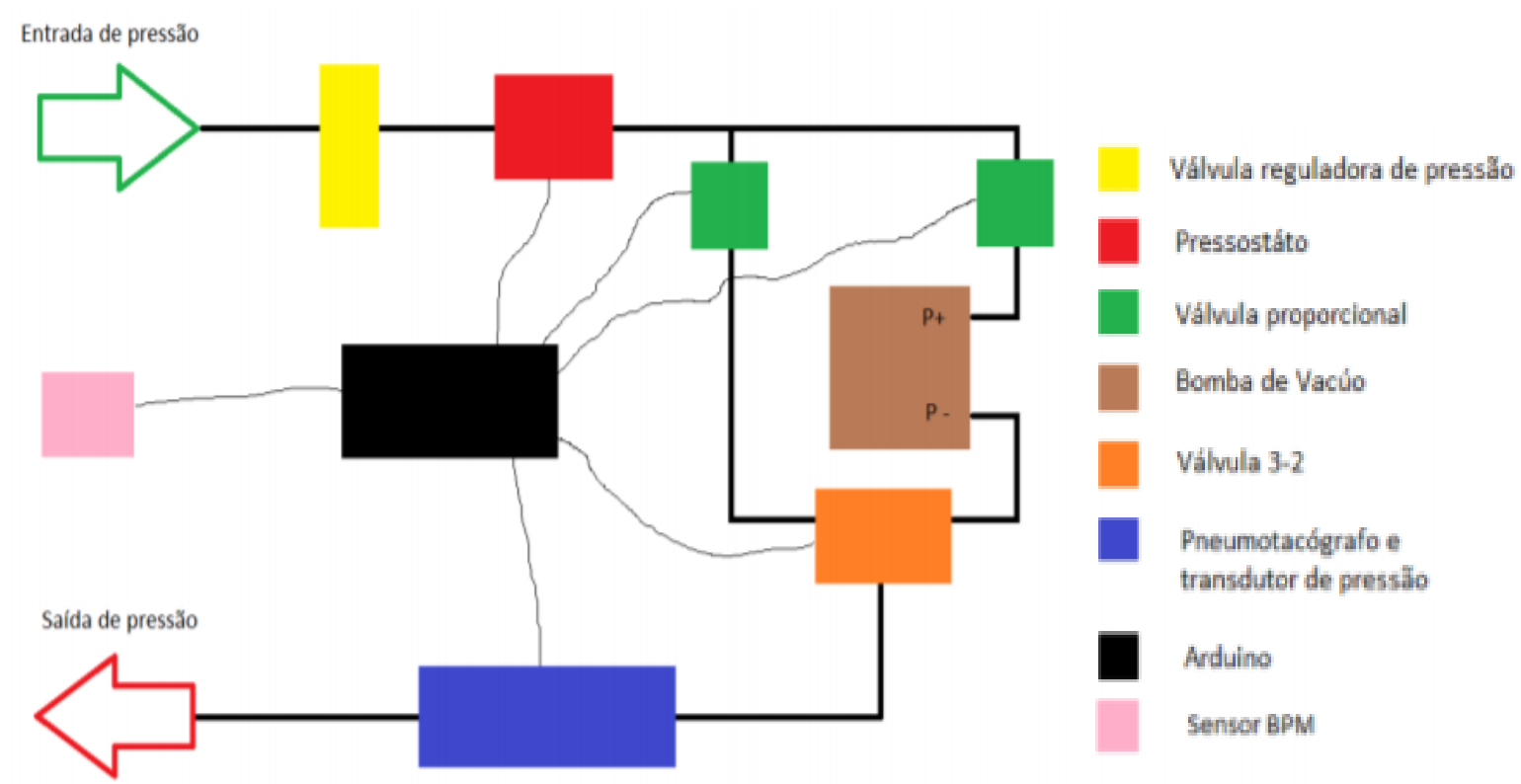

Figura 6 - Diagrama de controle com os componentes da bancada montada para os ensaios. 


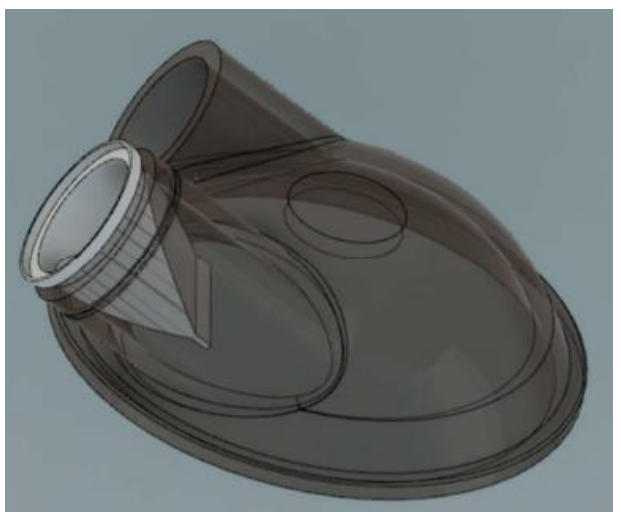

Figura 7 - Modelo tridimensional em CAD da câmara do dispositivo com detalhe da válvula.

Após a seleção do material e manufatura, uma nova etapa acontece que é a montagem do dispositivo e testes para a verificação da funcionalidade e validação do processo. Caso haja o retrabalho, é necessário utilizar limas tipo agulhas para a retirada de rebarbas, cantos vivos e ferimentos durante o manuseio [10].
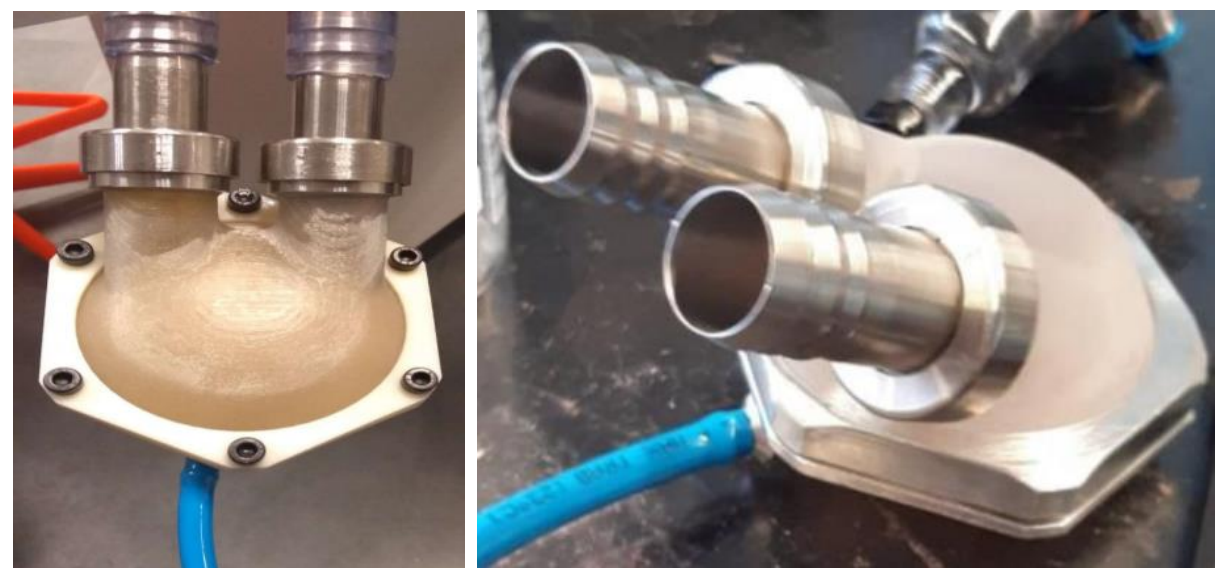

Figura 8 - Montagem do protótipo do coração artificial pneumático para os ensaios hidrodinâmicos. 


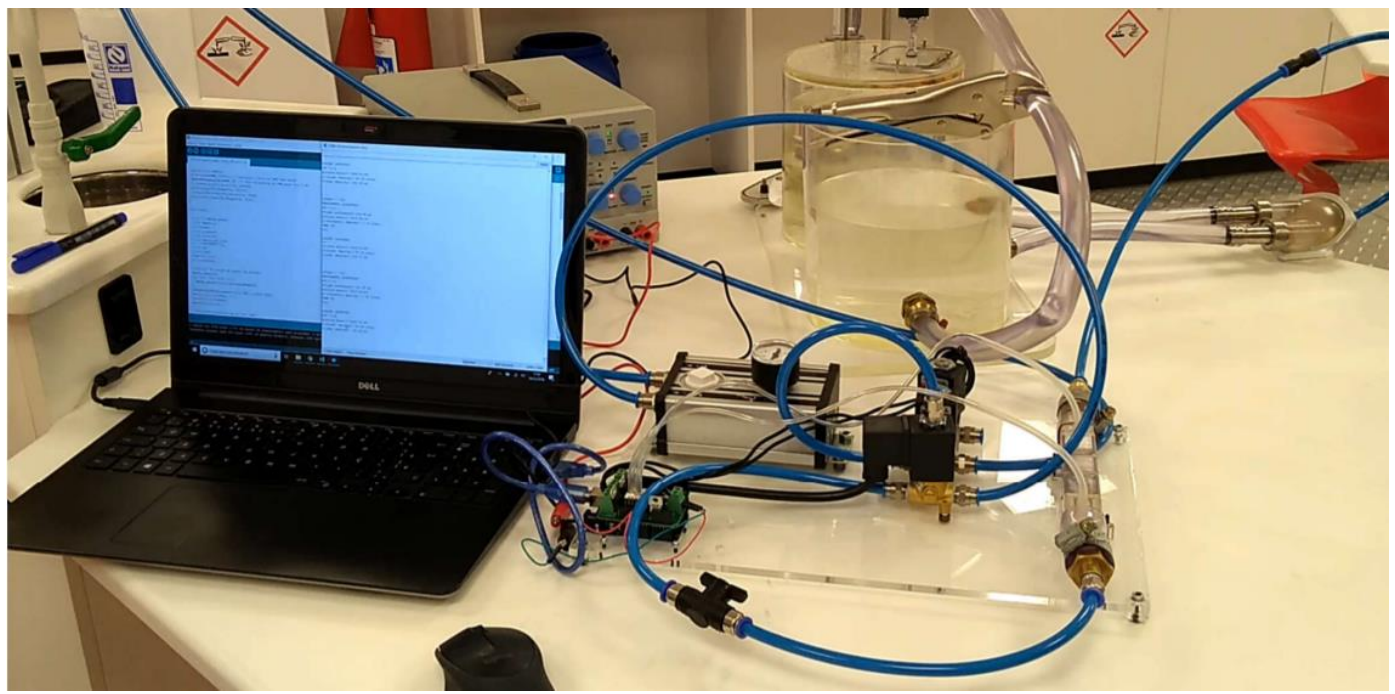

Figura 9 - Montagem da bancada de ensaios hidrodinâmicos e circuito de simulação do funcionamento.

Conclusão. Após a montagem do dispositivo o sensor de projeto foi calibrado na região da sístole e diástole para realização das tomadas de medição, garantindo a harmonia durante o funcionamento.

O desempenho do dispositivo (DAV) durante os teste realizados foram:

- 5 1/min de sangue contra uma pressão de $100 \mathrm{mmHg}$;

- Volume sistólico de $60 \mathrm{ml}$;

- Frequência cardíaca media de 80BPM para as condições de desempenho;

- Frequência cardíaca no interval de 30 a 130 BPM. 


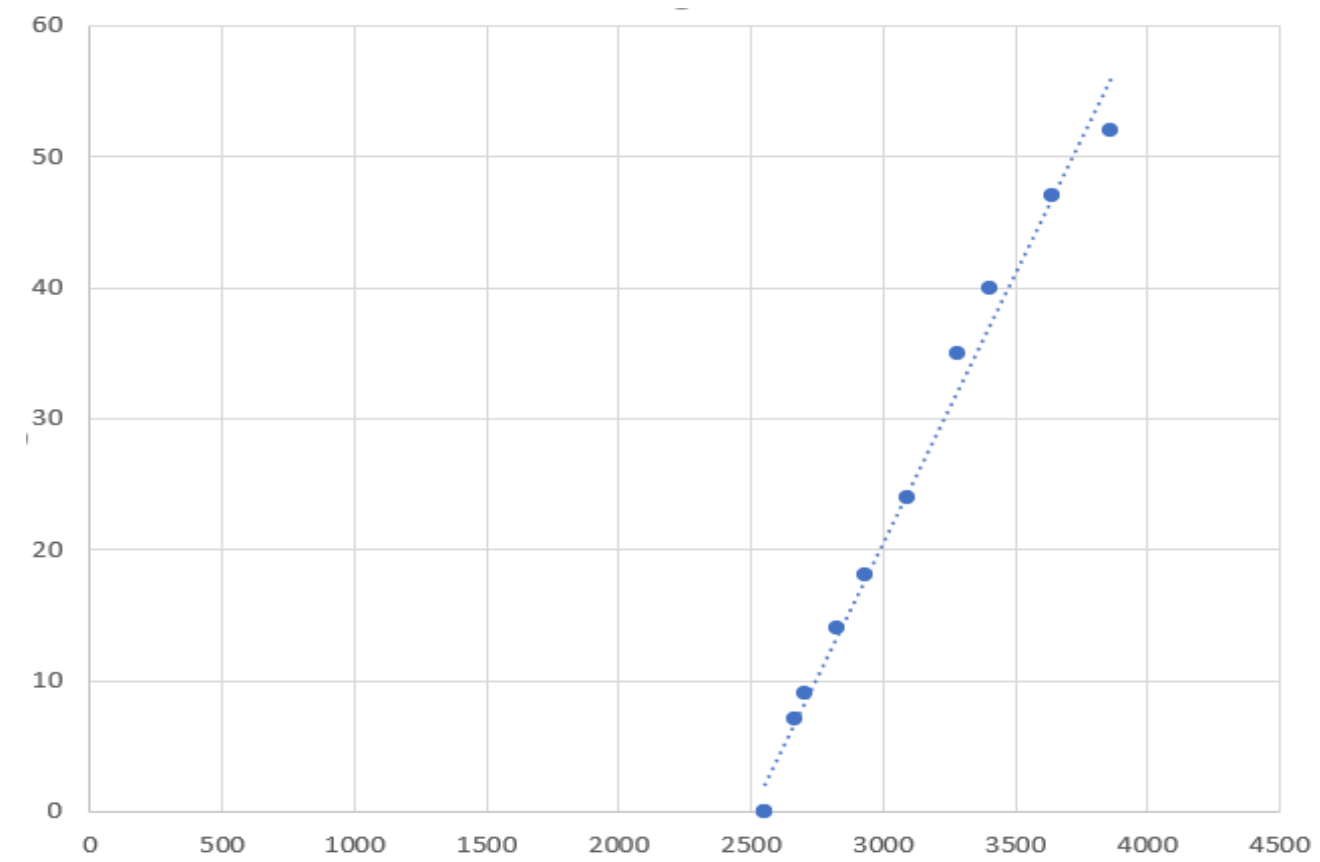

Figura 9 - Curva de calibração (tensão em mV) frente a vazão (1/min), sístole

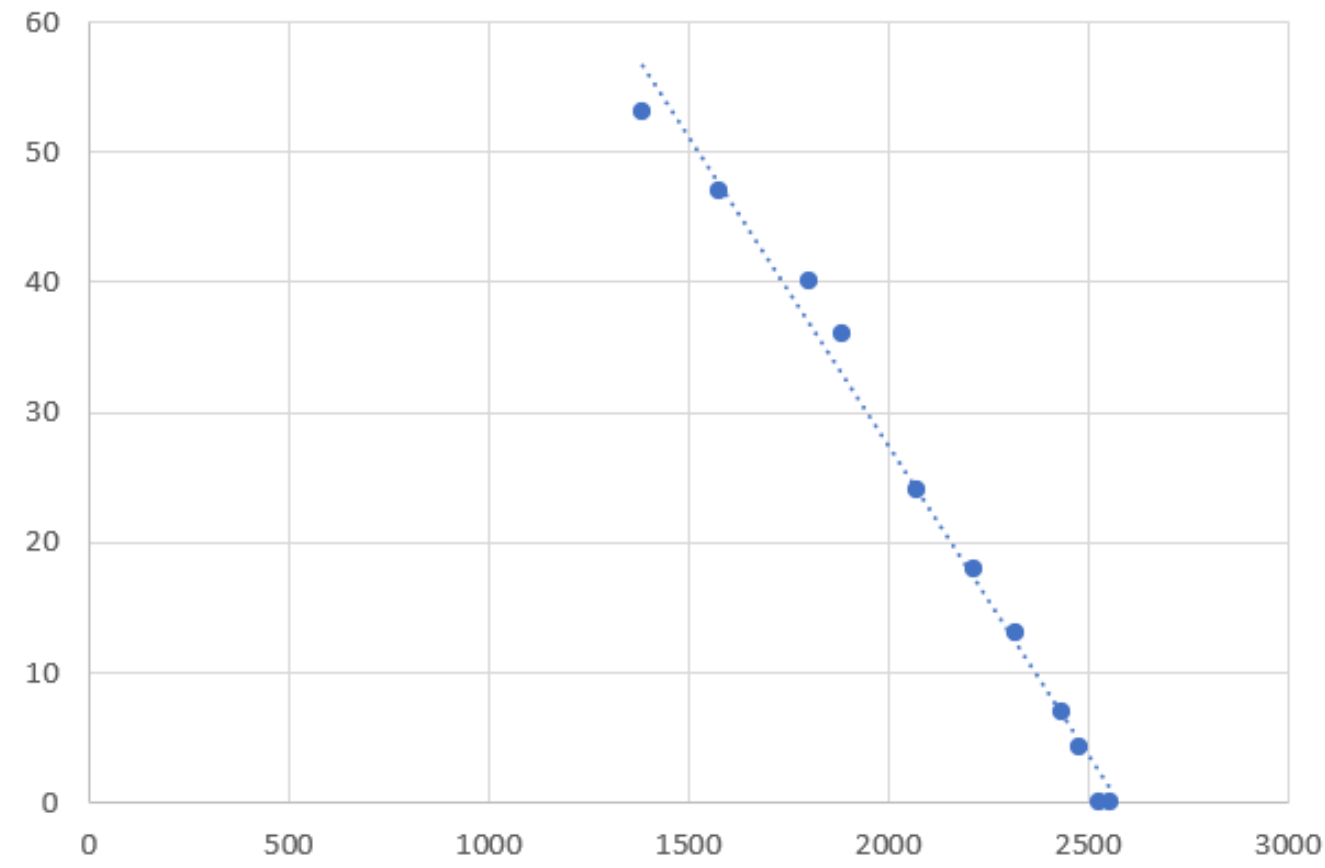

Figura 10 - Curva de calibração (tensão em mV) frente a vazão (1/min), diástole 
Comprovou-se, então, por intermédio da simulação numérica, que o dispositivo é hemocompatível no que tange ao fator interação dinâmica com o sangue. Para assegurar que o DAV fosse completamente hemocompatível, seria necessário realizar uma análise criteriosa dos materiais utilizados em todos componentes, avaliando de forma minuciosa a sua interação com o sangue.

Analisando os requisitos técnicos e a validação da bomba, é possível afirmar que o DAV projetado cumpriu os requisitos necessários. Este, com uma dimensão de 83.12 x 83.12 x $50 \mathrm{~mm}$, com uma vazão de 6,5L/min e um escoamento fisiológico, cumpriu os critérios esperados. Por mais que o coração artificial tenha apresentado um vazamento, este não foi significativo para prejudicar o seu funcionamento.

Agradecimentos. Agradeço ao Prof. Dr.Alex Camilli Bottene, coordenador do laboratório TechLab, a equipe técnica do laboratório do INSPER e ao Instituto Federal de São Paulo (IFSP) pelo o apoio.

\section{Referências}

(1) Ueberlhart B, Antunes PIT, Andrade AJP, Bock EG. Coração artificial e dispositivos de assistência circulatório no Brasil e no mundo. Revista Sinergia, 11(2), p151-155.

(2) Kormos RL, Cowger J, Pagani FD, Teuteberg JJ, Goldstein DJ, Jacobs JP, Higgins RS, Stevenson LW, Stehlik J, Atluri P, Grady KL, Kirklin JK. The Society of Thoracic Surgeons Intermacs Database Annual Report: Evolving Indications, Outcomes, and Scientific Partnerships. Ann Thor Surg 2019;107(2):341-353.

(3) Bock E, Ribeiro A, Silva M, Antunes P, Fonseca J, Legendre D, Leme J, Arruda C, Biscegli J, Nicolosi D, Andrade A. New Centrifugal Blood Pump With Dual Impeller and Double Pivot Bearing System: Wear Evaluation in Bearing System, Performance Tests, and Preliminary Hemolysis Tests. Artif Organs 2008;32(4):329-333.

(4) Hoshi H, Shinshi T, Takatani S. Third-generation Blood Pumps with Mechanical Noncontact Magnetic Bearings. Artif Organs, 2006;30(5):324-338.

(5) Sá RCL, Cruz NC, Moro JR, Leão TF, Andrade AJP, Bock EGP. Modification surface in medicine: techniques with Plasma in a Centrifugal Blood Pump Implantable, Sinergia, 2017; 18(2)91-94.

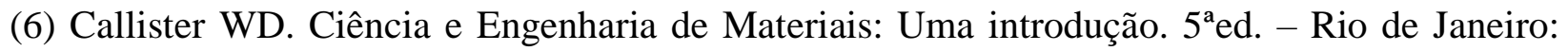
LTC, 2012. 
(7) Cestari VRF, Pessoa VLM, Moreira TMM, Florêncio RS, Barbosa IV, Ribeiro SB. Dispositivo de assistência ventricular e cuidados de enfermagem. Texto \& Contexto-Enfermagem , v.26, n.3, 2017.

(8) Groover, M.P. Fundamentos da Moderna Manufatura. 5aed. - Rio de Janeiro: LTC, 2017.

(9) Groover, M.P. Introdução aos Processos de Fabricação. 1ª ed. - Rio de Janeiro: LTC, 2014.

(10) Bock E, Leão T, Fonseca J, Andrade A. Left Ventricle Failure and Blood Flow Estimation for Centrifugal Blood Pumps. J Mech Eng Aut 2016;6:162-6. 